\title{
Estimation of Individual Cylinder Air-Fuel Ratio in Gasoline Engine with Output Delay
}

\author{
Changhui Wang and Zhiyuan Liu \\ Department of Control Science and Engineering, Harbin Institute of Technology, Harbin 150001, China \\ Correspondence should be addressed to Changhui Wang; wang_changhui@126.com
}

Received 1 March 2016; Revised 23 June 2016; Accepted 14 July 2016

Academic Editor: José A. Somolinos

Copyright (C) 2016 C. Wang and Z. Liu. This is an open access article distributed under the Creative Commons Attribution License, which permits unrestricted use, distribution, and reproduction in any medium, provided the original work is properly cited.

\begin{abstract}
The estimation of the individual cylinder air-fuel ratio (AFR) with a single universal exhaust gas oxygen (UEGO) sensor installed in the exhaust pipe is an important issue for the cylinder-to-cylinder AFR balancing control, which can provide high-quality torque generation and reduce emissions in multicylinder engine. In this paper, the system dynamic for the gas in exhaust pipe including the gas mixing, gas transport, and sensor dynamics is described as an output delay system, and a new method using the output delay system observer is developed to estimate the individual cylinder AFR. With the AFR at confluence point augmented as a system state, an observer for the augmented discrete system with output delay is designed to estimate the AFR at confluence point. Using the gas mixing model, a method with the designed observer to estimate the individual cylinder AFR is presented. The validity of the proposed method is verified by the simulation results from a spark ignition gasoline engine from engine software enDYNA by Tesis.
\end{abstract}

\section{Introduction}

Cylinder-to-cylinder air-fuel ratio (AFR) balancing control in internal combustion engines with multiple cylinders is one of the technology trends to satisfy the increasingly stringent emission regulations, which can also improve the engine performance, such as thermal efficiency and fuel economy. The AFR of each cylinder is decided by the aspirated air mass, the injected fuel mass, and the residual gas from the prior cycle, in which the combustion stroke of each cylinder sequentially occurs along the rotational angle of the crankshaft. Due to the air breathing variability and injector variability, there exists AFR imbalance between cylinders, leading to adverse impacts on emission performance using the conventional controllers [1,2].

In order to improve the AFR control accuracy, there has been a great deal of research that focuses on the AFR control of individual cylinders [3-10]. In fact, the estimation of the individual cylinder AFR with a single universal exhaust gas oxygen (UEGO) sensor installed in the exhaust pipe is one of the key technology trends for the individual cylinder AFR control. The digital filtering techniques are employed to extract the AFR imbalance signals from oxygen sensor voltage signals in [11], in which the oxygen sensor voltage signal is processed to determine imbalanced cylinder identification and AFR cylinder imbalance levels. In [12], a modeling method to estimate the individual fuel-gas ratio is proposed to estimate AFR, which is used for an adaptive generalized predictive control approach to balance the individual cylinder characteristics in the static engine operation mode. A static steady state observer based on the individual cylinder AFR model along the air mass flow, gas mixing, and sensor dynamics in an exhaust manifold can be found in [13]. In the diesel engines, a nonlinear observer is proposed to estimate the individual cylinder AFR [14]. In [15], a PI compensator is designed to compensate the cylinder-by-cylinder variations, in which an input observer is proposed to estimate individual cylinder AFR.

However, the transport delay and sensor delay from the exhaust confluence point to UEGO sensor output are ignored in the proposed observers from the above papers, which may reduce the accuracy of the AFR estimation of each cylinder. In order to improve the individual cylinder AFR estimation accuracy, the system dynamics in the exhaust pipe including gas transport and sensor dynamics is described as an augmented discrete system with output delay in this paper, in which the AFR at confluence point is augmented as 


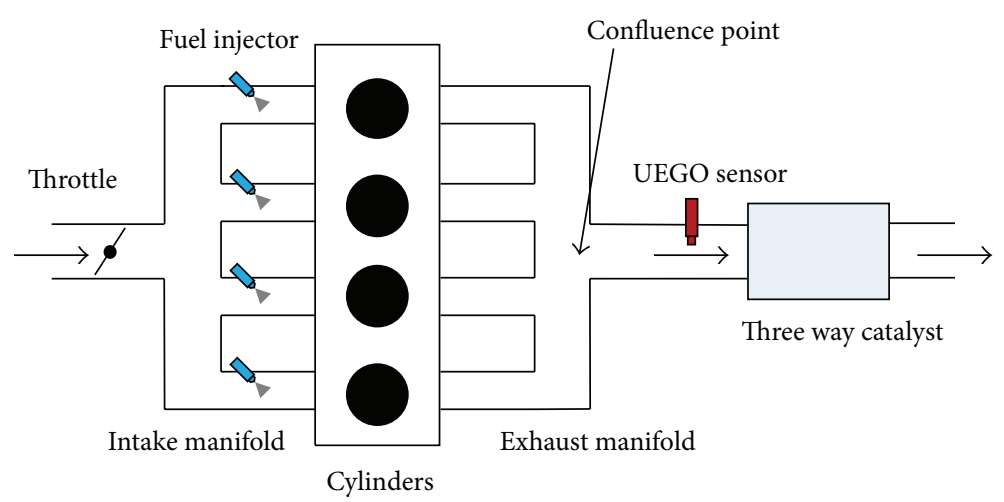

FIgURE 1: Schematic of the 4-cylinder SI gasoline engine.

a system state. Then, an observer for the augmented discrete system with output delay is designed. With the combination of the designed observer and the gas mixing model at confluence point, the method to estimate the individual cylinder AFR is presented. The performance of the proposed method is validated against the simulation result from engine software enDYNA provided by Tesis, and a comparison with existing method is given during an urban driving cycle, which demonstrates that the proposed method can improve the accuracy of the individual cylinder AFR estimation.

This paper is organized as follows. In Section 2, the system dynamics in the exhaust pipe including gas transport and UEGO sensor dynamics is described as an augmented system with output delay. In Section 3, an observer for the output delay system is designed, and the method to estimate the individual cylinder AFR is presented. Simulation results from enDYNA are presented in Section 4, and the conclusions are summarized in Section 5.

\section{Problem Formulation}

A schematic diagram of a 4-cylinder spark-ignited (SI) gasoline engine is shown in Figure 1, where the fuel injectors equipped at the inlet port near to the intake valve are controlled individually. The fuel mass burnt in each cylinder is injected by the corresponding injector, and the fuel injection command is delivered to the injector of each cylinder serially along the crank angle. The AFR of each cylinder is $\lambda_{i}=$ $m_{\text {cyli }} / m_{\text {air }}, i=1,2,3,4$, where $m_{\text {cyli }}$ is the fuel mass into the cylinder and $m_{\text {air }}$ is the air mass into the cylinder.

After combustion, the combusted gas of each cylinder is exhausted into the corresponding runner during the exhaust stroke of each cylinder and passes through their runners and confluence in the public exhaust manifold. Then, the AFR of the mixed gas is measured by a UEGO sensor, and the mixed gas runs to the outside passing through the catalyst. The system dynamics in the exhaust pipe includes the gas mixing, gas transport, and sensor dynamics, in which the transfer function from the confluence point to sensor output can be given by [13]

$$
G_{\mathrm{exh}}(s)=\frac{\lambda_{\mathrm{sen}}(s)}{\lambda_{c}(s)}=\frac{e^{-\delta s}}{\left(1+\tau_{\mathrm{mix}} s\right)\left(1+\tau_{\mathrm{sen}} s\right)},
$$

where $\lambda_{c}$ is the AFR at the exhaust confluence point, $\lambda_{\text {sen }}$ is the measured AFR of the UEGO sensor, $\delta=\delta_{\text {mix }}+\delta_{\text {sen }}$ is the time delay including the transport delay $\delta_{\text {mix }}$ and sensor delay $\delta_{\text {sen }}, \tau_{\text {mix }}$ is the time constant of the mixing process, and $\tau_{\text {sen }}$ is the sensor time constant.

For an engine with 4 cylinders, the combined UEGO sensor signal is sampled at the exhaust top dead center of each cylinder, and the sampling period $T_{s}$ is related to the engine cycle period as $T_{s}=T_{c} / 4$, in which the engine cycle period is $T_{c}=120 / n_{e}$, where $n_{e}$ is the engine speed in rpm. Consisting of the zero-order holder and the mixing and sensor dynamics, the discrete form of model (1) with the sampling period $T_{s}$ can be given by [13]

$$
Q_{\mathrm{exh}}(z)=z^{-m}\left(1+a \frac{z-1}{z-\alpha_{\text {mix }}}+b \frac{z-1}{z-\alpha_{\text {sen }}}\right)
$$

where

$$
\begin{aligned}
\alpha_{\text {mix }} & =e^{-T_{s} / \tau_{\text {mix }},} \\
\alpha_{\text {sen }} & =e^{-T_{s} / \tau_{\text {sen }}}, \\
m & =\frac{\delta}{T_{s}}, \\
a & =\frac{-\tau_{\text {mix }}}{\tau_{\text {mix }}-\tau_{\text {sen }}}, \\
b & =\frac{\tau_{\text {sen }}}{\tau_{\text {mix }}-\tau_{\text {sen }}}, \\
z & =e^{T_{s} s} .
\end{aligned}
$$

Furthermore, (2) can be written as a state-space form with input delay [15]:

$$
\begin{aligned}
x_{q}(l+1) & =A_{q} x_{q}(l)+B_{q} \lambda_{c}(l-m), \\
\lambda_{\text {sen }}(l) & =C_{q} x_{q}(l),
\end{aligned}
$$


where

$$
\begin{aligned}
& A_{q}=\left(\begin{array}{cc}
\alpha_{\text {mix }} & 0 \\
0 & \alpha_{\text {sen }}
\end{array}\right), \\
& B_{q}=\left(\begin{array}{l}
1 \\
1
\end{array}\right) \text {, } \\
& C_{q}=\left(a\left(\alpha_{\text {mix }}-1\right) b\left(\alpha_{\text {sen }}-1\right)\right), \\
& x_{q}(l)=\left(\begin{array}{c}
x_{q 1}(l) \\
x_{q 2}(l)
\end{array}\right), \\
& x_{q 1}(l)=\frac{\lambda_{\text {sen }}(l)-b\left(\alpha_{\text {sen }}-1\right) x_{q 2}(l)}{a\left(\alpha_{\text {mix }}-1\right)}, \\
& x_{q 2}(l)=\frac{1}{\left(\alpha_{\text {sen }}-\alpha_{\text {mix }}\right) b\left(\alpha_{\text {sen }}-1\right)}\left(\alpha_{\text {sen }} \lambda_{\text {sen }}(l)\right. \\
& -\alpha_{\text {mix }} \alpha_{\text {sen }} \lambda_{\text {sen }}(l-1) \\
& +\left(1-2 a \alpha_{\text {sen }}-2 b \alpha_{\text {mix }}-\alpha_{\text {mix }}-\alpha_{\text {sen }}+a+b\right) \\
& \cdot \lambda_{c}(l-m)+\left(a \alpha_{\text {sen }}+b \alpha_{\text {mix }}+\alpha_{\text {mix }} \alpha_{\text {sen }}\right) \\
& \left.\cdot \lambda_{c}(l-m-1)\right) \text {. }
\end{aligned}
$$

Define the new system state as $\bar{x}_{q}(l)=x_{q}(l+m)$, and (4) with input delay can also be rewritten as the following discrete system with output delay:

$$
\begin{aligned}
\bar{x}_{q}(l+1) & =A_{q} \bar{x}_{q}(l)+B_{q} \lambda_{c}(l), \\
\lambda_{\text {sen }}(l) & =C_{q} \bar{x}_{q}(l-m) .
\end{aligned}
$$

$\lambda_{c}(l)$ in (6) is unknown, which can be considered as a system state due to the small rate of change of $\lambda_{c}(l)$. Then, an augmented discrete system with output delay from (6) can be obtained:

$$
\begin{aligned}
x(l+1) & =A x(l), \\
y(l) & =C x(l-m),
\end{aligned}
$$

where

$$
\begin{aligned}
x(l) & =\left(\begin{array}{l}
\bar{x}_{q}(l) \\
\lambda_{c}(l)
\end{array}\right), \\
A & =\left(\begin{array}{ccc}
\alpha_{\text {mix }} & 0 & 1 \\
0 & \alpha_{\text {sen }} & 1 \\
0 & 0 & 1
\end{array}\right), \\
y(l) & =\lambda_{\text {sen }}(l), \\
C & =\left(\begin{array}{lll}
a\left(\alpha_{\text {mix }}-1\right) & b\left(\alpha_{\text {sen }}-1\right) & 0
\end{array}\right) .
\end{aligned}
$$

Equation (7) indicates that the estimation of the AFR $\lambda_{c}$ at the exhaust confluence point becomes the state estimation of the discrete system with output delay (7).

\section{Observer Design for Discrete System with Output Delay}

The observer for the output delay system (7) is given:

$$
\begin{aligned}
\widehat{x}(l+1) & =A \widehat{x}(l)+L(y(l)-C \widehat{x}(l-m)), \\
\hat{y}(l) & =C \widehat{x}(l-m),
\end{aligned}
$$

where $\hat{x} \in \mathbb{R}$ is the state estimate and $L \in \mathbb{R}^{3 \times 1}$ is the feedback gain matrix. The asymptotical stability of the proposed observer (9) is analyzed in the following theorem.

Theorem 1. There exist matrices $L, P=P^{T}>0, Q=Q^{T} \geq 0$, and $Z=Z^{T} \geq 0$, such that the following linear matrix inequality (LMI) is feasible:

$$
\Xi=\left(\begin{array}{ccccc}
-P+Q+M^{T}+M & -M^{T}+N & A^{T} P & M^{T} & \left(A^{T}-I\right) Z \\
-M+N^{T} & -Q-N^{T}-N & -C^{T} L^{T} P & N^{T} & -C^{T} L^{T} Z \\
P A & -P L C & -P & 0 & 0 \\
M & N & 0 & -m^{-1} Z & 0 \\
Z(A-I) & -Z L C & 0 & 0 & m^{-1}(Z-2 P)
\end{array}\right)<0
$$

then observer (9) is asymptotically stable.

Denote $\eta(l)=e(l+1)-e(l)$; then we have

Proof. Set the estimation error $e(l)=\widehat{x}(l)-x(l)$, and the error dynamic system between (7) and (9) is obtained:

$$
\begin{aligned}
e(l-m) & =e(l)-\sum_{n=l-m}^{l-1} \eta(n), \\
\eta(l) & =(A-I) e(l)-L C e(l-m) .
\end{aligned}
$$

$$
e(l+1)=A e(l)-L C e(l-m) .
$$


Choose a Lyapunov functional candidate as

$$
\begin{aligned}
V(l)= & e^{T}(l) P e(l)+\sum_{i=l-m}^{l-1} e^{T}(i) Q e(i) \\
& +\sum_{j=-m+1}^{0} \sum_{i=l-1+j}^{l-1} \eta^{T}(i) Z \eta(i) .
\end{aligned}
$$

Define $\Delta V=V(l+1)-V(l)$; then along the solution of (11) and (12) we have

$$
\begin{aligned}
& \Delta V(l)=\left(\begin{array}{c}
e(l) \\
e(l-m)
\end{array}\right)^{T} \\
& \cdot\left(\begin{array}{cc}
-P+Q+A^{T} P A & -A^{T} P L C \\
-C^{T} L^{T} P A & -Q+C^{T} L^{T} P L C
\end{array}\right)\left(\begin{array}{c}
e(l) \\
e(l-m)
\end{array}\right) \\
& +m \eta^{T}(l) Z \eta(l)-\sum_{n=l-m}^{l-1} \eta^{T}(n) Z \eta(n) .
\end{aligned}
$$

According to Lemma 1 in [16], for any constant matrix $Z>0$, $M$, and $N$, the following inequality holds:

$$
\begin{gathered}
-\sum_{n=l-m}^{l-1} \eta^{T}(n) Z \eta(n) \leq\left(\begin{array}{c}
e(l) \\
e(l-m)
\end{array}\right)^{T} \\
\cdot\left(\begin{array}{cc}
M^{T}+M & -M^{T}+N \\
-M+N^{T} & -N^{T}-N
\end{array}\right)\left(\begin{array}{c}
e(l) \\
e(l-m)
\end{array}\right) \\
+m\left(\begin{array}{c}
e(l) \\
e(l-m)
\end{array}\right)^{T} \\
\cdot\left(\begin{array}{c}
M^{T} \\
N^{T}
\end{array}\right) Z^{-1}\left(\begin{array}{ll}
M & N
\end{array}\right)\left(\begin{array}{c}
e(l) \\
e(l-m)
\end{array}\right) .
\end{gathered}
$$

With the combination of (14) and (15), we have

$$
\Delta V(l) \leq\left(\begin{array}{c}
e(l) \\
e(l-m)
\end{array}\right)^{T} \Omega\left(\begin{array}{c}
e(l) \\
e(l-m)
\end{array}\right)
$$

where

$\Omega$

$$
=\left(\begin{array}{cc}
-P+Q+M^{T}+M+A^{T} P A+m(A-I)^{T} Z(A-I)+m M^{T} Z^{-1} M & -M^{T}+N-A^{T} P L C-m(A-I)^{T} Z L C+m M^{T} Z^{-1} N \\
* & -Q-N^{T}-N+C^{T} L^{T} P L C-m C^{T} L^{T} Z L C+m N^{T} Z^{-1} N
\end{array}\right) .
$$

By Schur complement [17], the following LMI (18) guarantees $\Omega<0$, which can guarantee $\Delta V(l)<0$ and the asymptotical stability of observer (9):

$$
\Pi=\left(\begin{array}{ccccc}
-P+Q+M^{T}+M & -M^{T}+N & A^{T} P & M^{T} & \left(A^{T}-I\right) Z \\
-M+N^{T} & -Q-N^{T}-N & -C^{T} L^{T} P & N^{T} & -C^{T} L^{T} Z \\
P A & -P L C & -P & 0 & 0 \\
M & N & 0 & -m^{-1} Z & 0 \\
Z(A-I) & -Z L C & 0 & 0 & -m^{-1} Z
\end{array}\right)<0
$$

Now, condition (10) guaranteeing (18) must be proved. Define $W=\operatorname{diag}\left(I, I, I, I, P Z^{-1}\right)$, and we have

$$
W^{T} \Pi W=\left(\begin{array}{ccccc}
-P+Q+M^{T}+M & -M^{T}+N & A^{T} P & M^{T} & \left(A^{T}-I\right) P \\
-M+N^{T} & -Q-N^{T}-N & -C^{T} L^{T} P & N^{T} & -C^{T} L^{T} P \\
P A & -P L C & -P & 0 & 0 \\
M & N & 0 & -m^{-1} Z & 0 \\
P(A-I) & -P L C & 0 & 0 & -m^{-1} P Z^{-1} P
\end{array}\right) .
$$




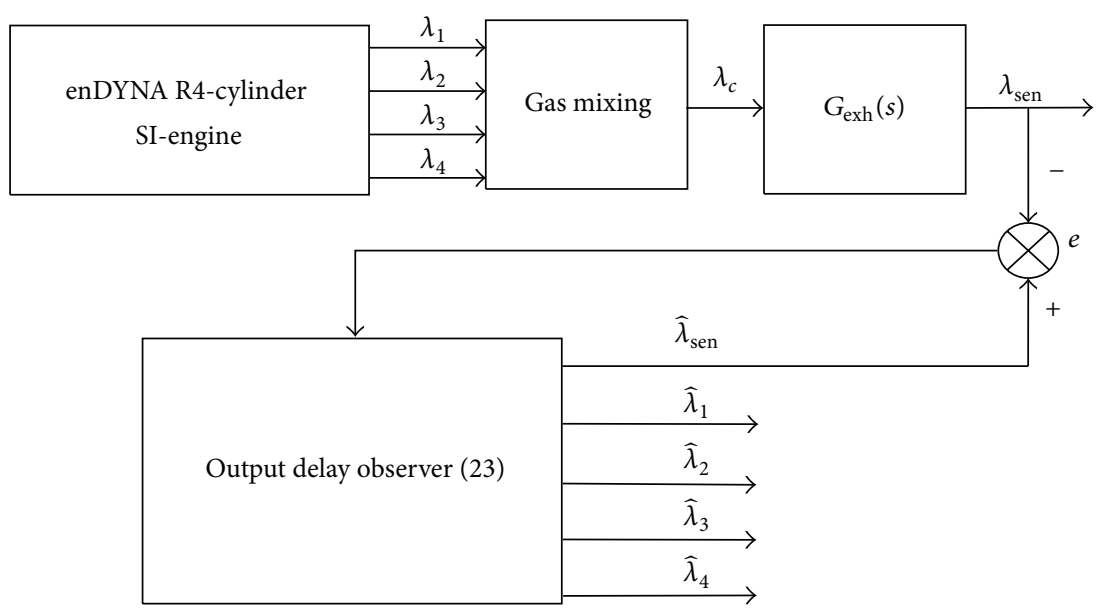

FIGURE 2: Schematic diagram for the estimation of the individual cylinder AFR.

Because of the fact that $(P-Z) Z^{-1}(P-Z) \geq 0$, we have $-P Z^{-1} P \leq Z-2 P$. Therefore, condition (10) can guarantee $W^{\mathrm{T}} \Pi W<0$; then (18) holds.

The estimation of the AFR $\lambda_{c}$ at the exhaust confluence point can be obtained according to observer (9). In order to obtain the AFR of each cylinder, the relationship between the AFR of each cylinder and the AFR at the exhaust confluence point is analyzed in the following.

The combusted gas of each cylinder is discharged into the corresponding exhaust port and flows to the exhaust confluence point in the exhaust manifold, in which we assume that exhaust gas mixing in the individual exhaust runner can be neglected. Hence, the AFR in the exhaust runner is constant during one engine cycle, and the AFR $\lambda_{c}$ at the exhaust confluence point in the $k$ th engine cycle can be given by [15]

$$
\begin{aligned}
\lambda_{c}\left(k T_{c}+(i-1) T_{s}\right) & \\
= & \frac{\sum_{j=1}^{i} \dot{m}_{c j}\left(k T_{c}+(i-1) T_{s}\right) \lambda_{j}(k)}{\sum_{l=1}^{4} \dot{m}_{c l}\left(k T_{c}+(i-1) T_{s}\right)} \\
& +\frac{\sum_{j=1}^{4} \dot{m}_{c j}\left(k T_{c}+(i-1) T_{s}\right) \lambda_{j}(k-1)}{\sum_{l=1}^{4} \dot{m}_{c l}\left(k T_{c}+(i-1) T_{s}\right)},
\end{aligned}
$$

where $\dot{m}_{c i}$ is the exhaust air mass flow in the $i$ th exhaust manifold passing through the confluence point and $\lambda_{i}$ is the AFR in the ith cylinder. Furthermore, under the assumption that air mass aspirated into cylinders in each cycle is constant, exhaust air flow has the same shape between successive cycles; then a periodic function can be obtained:

$$
\begin{aligned}
\gamma_{i j}(k) & =\frac{\dot{m}_{c j}\left(k T_{c}+(i-1) T_{s}\right)}{\sum_{l=1}^{4} \dot{m}_{c l}\left(k T_{c}+(i-1) T_{s}\right)} \\
& =\frac{\dot{m}_{c j}\left((i-1) T_{s}\right)}{\sum_{l=1}^{4} \dot{m}_{c l}\left((i-1) T_{s}\right)} .
\end{aligned}
$$

Therefore, the gas mixing behavior (20) can be rewritten in the $T_{s}$ domain as

$$
\lambda_{c}(l)=\sum_{i=0}^{3} \gamma_{[l][l-i]} \lambda_{[l-i]}(l-i)
$$

where $[l]=(i \bmod 4)+1$. The relationship between the AFR of each cylinder and the AFR at the exhaust confluence point can be obtained by (22).

The algorithm to estimate the individual cylinder AFR is as follows: First, the AFR $\lambda_{c}$ at the exhaust confluence point is obtained according to observer (9). Then, the individual cylinder AFR can be calculated through (22). With the combination of (9) and (22), the method for the estimation of each cylinder AFR can be given as follows:

$$
\begin{aligned}
& \widehat{x}(l+1)=A \hat{x}(l)+L(y(l)-C \widehat{x}(l-m)), \\
& \hat{\lambda}_{c}(l)=\left(\begin{array}{lll}
0 & 0 & 1
\end{array}\right) \cdot \hat{x}(l), \\
& \hat{\lambda}_{[l]}(l)=\frac{1}{\gamma_{[l][l]}}\left(-\sum_{i=1}^{3}\left(\gamma_{[l][l-i]} \cdot \hat{\lambda}_{[l-i]}(l-i)\right)+\hat{\lambda}_{c}(l)\right) .
\end{aligned}
$$

\section{Simulation Studies}

In this section, the simulation study of the estimation of the individual cylinder AFR is presented in the environment of a 2.0 L 4-cylinder SI gasoline engine from enDYNA $[18,19]$. The enDYNA is a professional software tool for the real-time simulation of internal combustion engines, providing readyto-use models for all common engine types comprising crank angle synchronous combustion, gas path, fuel system, cooling system, drivetrain, driver, and soft-ECU. The R4-cylinder SIengine is an example in enDYNA to simulate a 4-cylinder SI gasoline engine, whose specifications are given in Table 1 . The observer architecture is illustrated in Figure 2. 

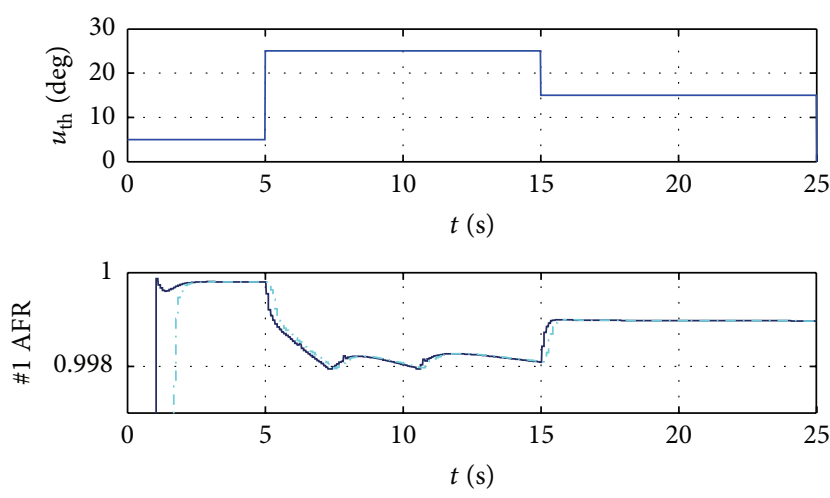

— enDYNA

- Estimated
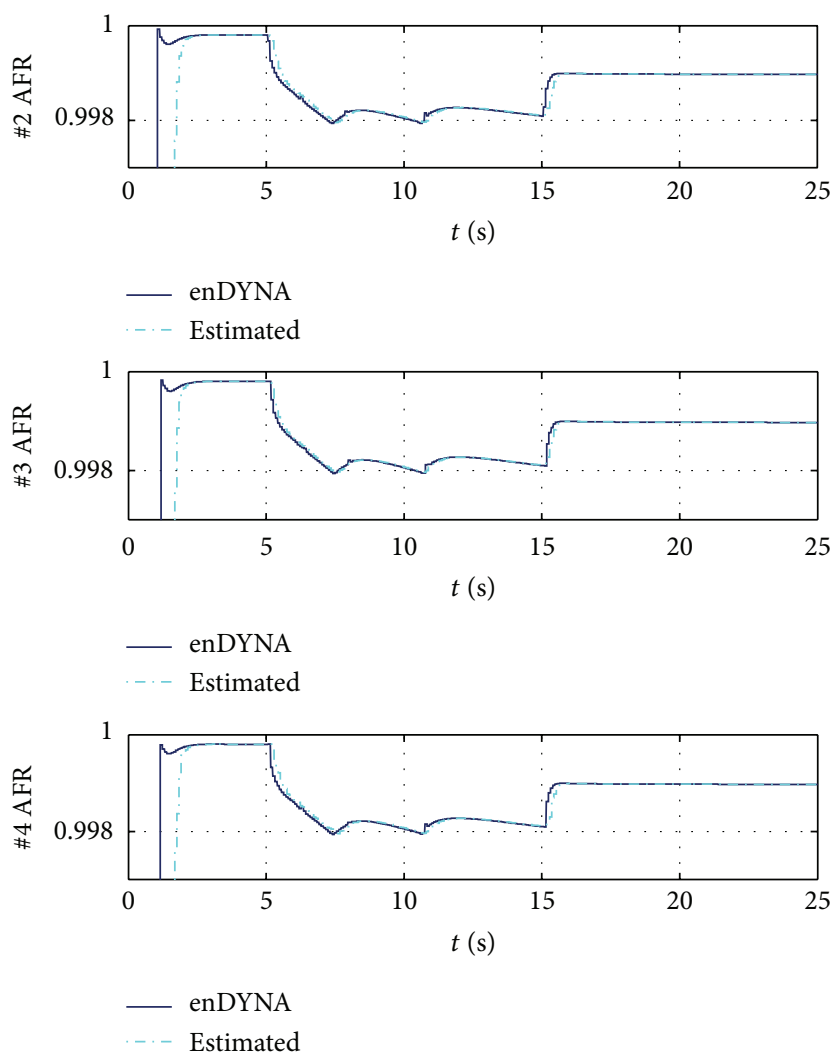

FIGURE 3: Individual cylinder AFR estimation results.

TABLE 1: Engine specifications.

\begin{tabular}{lc}
\hline Fuel system & Direct injection \\
\hline Displacement $\left(\mathrm{m}^{3}\right)$ & 0.002 \\
Intake manifold volume $\left(\mathrm{m}^{3}\right)$ & 0.004 \\
Exhaust manifold volume $\left(\mathrm{m}^{3}\right)$ & 0.0015 \\
Max engine speed $(\mathrm{rad} / \mathrm{s})$ & 785 \\
\hline
\end{tabular}

The parameters of the system dynamics in the exhaust pipe (2) and the gas mixing (22) are presented as follows [15]:

$$
\begin{aligned}
& \tau_{\text {mix }}=0.01 \mathrm{~s}, \\
& \tau_{\text {sen }}=0.12 \mathrm{~s},
\end{aligned}
$$
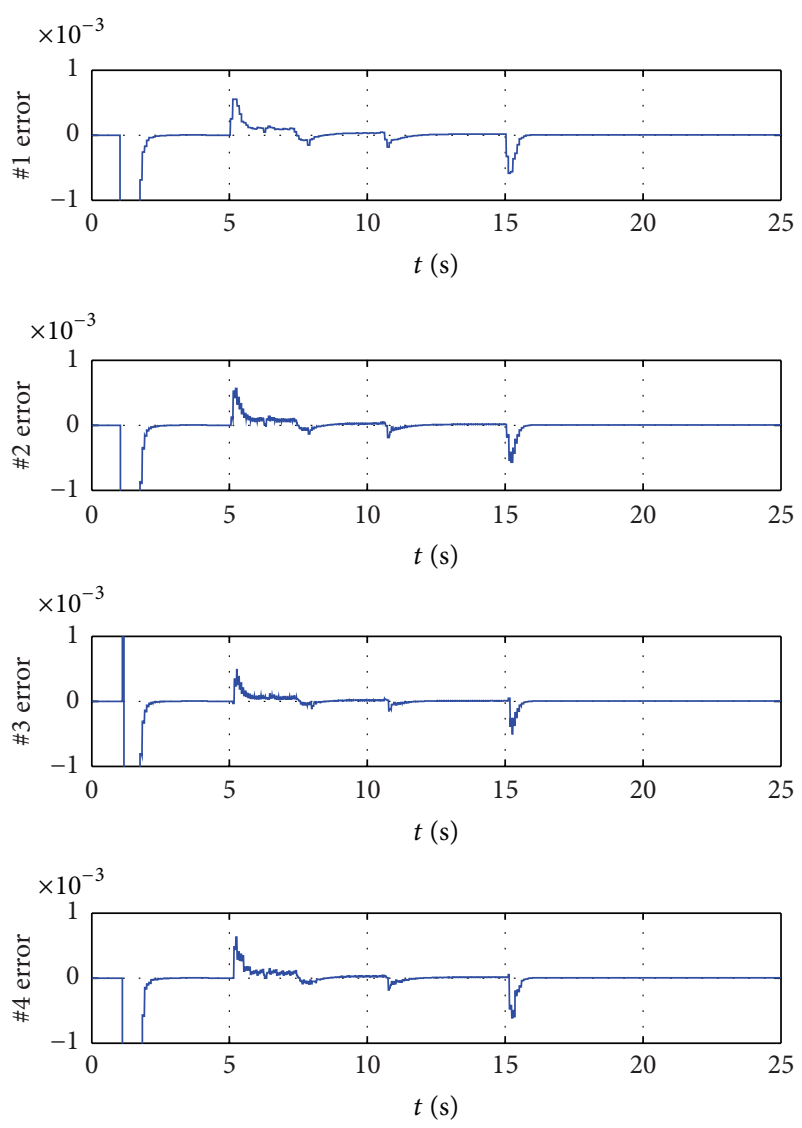

FIGURE 4: Evolution of the estimation error.

$$
\begin{aligned}
m & =1, \\
T_{c} & =0.08 \mathrm{~s}, \\
T_{s} & =0.02 \mathrm{~s}, \\
\Gamma & =\left(\begin{array}{cccc}
0.55 & 0.05 & 0.15 & 0.25 \\
0.25 & 0.6 & 0.05 & 0.1 \\
0.15 & 0.25 & 0.5 & 0.1 \\
0.05 & 0.1 & 0.15 & 0.7
\end{array}\right) .
\end{aligned}
$$

Then, the system matrix can be obtained:

$$
\begin{aligned}
& A=\left(\begin{array}{ccc}
0.1353 & 0 & 1 \\
0 & 0.8465 & 1 \\
0 & 0 & 1
\end{array}\right), \\
& C=\left(\begin{array}{lll}
-0.0786 & 0.1675 & 0
\end{array}\right) .
\end{aligned}
$$

According to the inequality (10), the gain matrix can be given by $L=\left(\begin{array}{lll}0.97 & 3.79 & 0.73\end{array}\right)^{T}$.

Here, the input of the throttle angle in enDYNA is designed as a step signal presented in Figure 3. Accordingly, the estimation results of the individual cylinder AFR by the proposed method are shown in Figure 3, and the estimation errors are plotted in Figure 4. When the throttle changes 

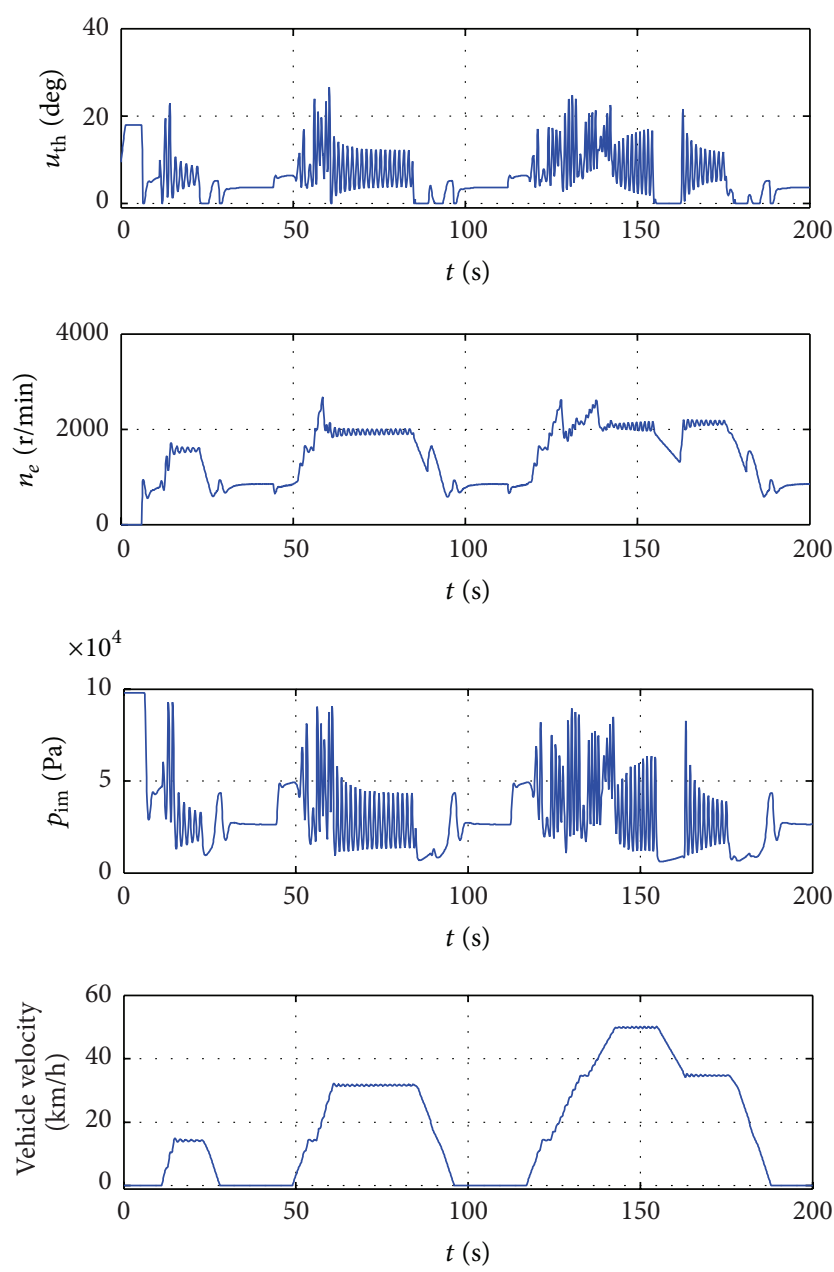

FIGURE 5: Evolution of throttle angle $u_{\text {th }}$, engine speed $n_{e}$, intake manifold pressure $p_{\mathrm{im}}$, and vehicle velocity under ECE cycle.

suddenly, the estimation errors of the individual cylinder AFR are about $0.06 \%$, and then the steady state errors tend to decay within $0.01 \%$.

In order to verify the effectiveness of the proposed method under driving cycle condition, one segment of the urban driving cycle ECE (Economic Commission for Europe) is used [19], under which the throttle angle $u_{\text {th }}$, engine speed $n_{e}$, intake manifold pressure $p_{\text {im }}$, and vehicle velocity are plotted in Figure 5. Accordingly, the comparison of the individual cylinder AFR estimation between the proposed method and the input observer in [15] are presented in Figure 6, and the estimation errors are plotted in Figure 7. Clearly, the error of the proposed method is smaller than the input observer when the AFR changes slowly, in which the steady state error of the input observer is $0.03 \%$. When the AFR changes severely, there exist fluctuations of the AFR estimation error from both the proposed method and input observer in [15]. However, the estimation error from the proposed method is smaller. It is demonstrating that the proposed method considering time delay can improve the accuracy of the individual cylinder AFR estimation.
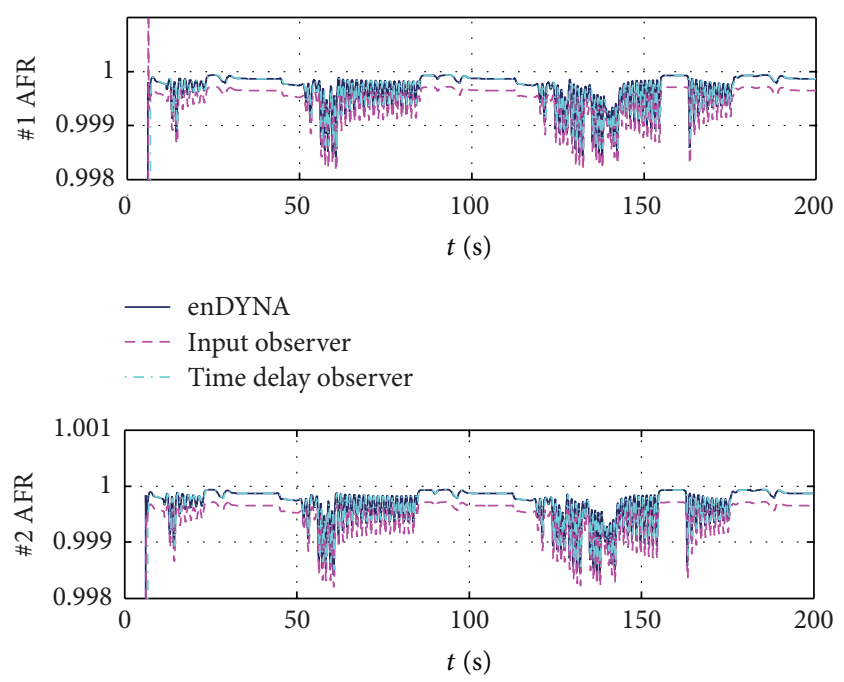

- enDYNA

- - - Input observer

-. - Time delay observer

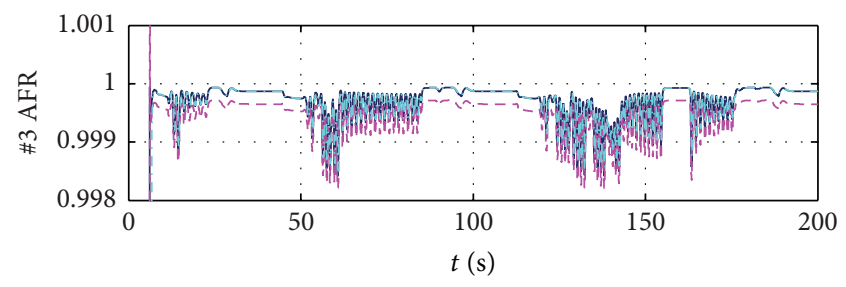

- enDYNA

- - - Input observer

- - Time delay observer

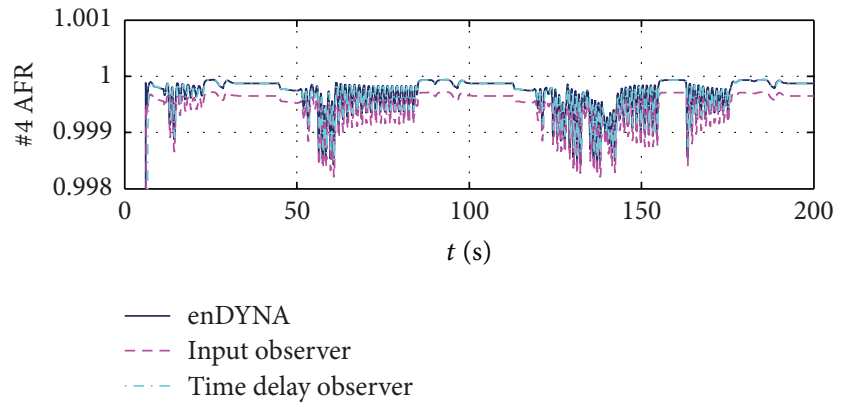

FIgURE 6: Individual cylinder AFR estimation results of the proposed method and unknown input observer under ECE cycle.

\section{Conclusion}

An efficient method for the estimation of the individual cylinder AFR with a single UEGO sensor was developed to improve the estimation accuracy. The system dynamics in the exhaust pipe was described as an augmented discrete system with output delay, in which the AFR at confluence point was augmented as a system state and beneficial to be estimated comparing the system with input delay. Then, an observer for the augmented system with output delay was designed to estimate the AFR at confluence point, which can avoid accurately inverting the engine model including delays. Using the gas mixing model, a method to estimate 


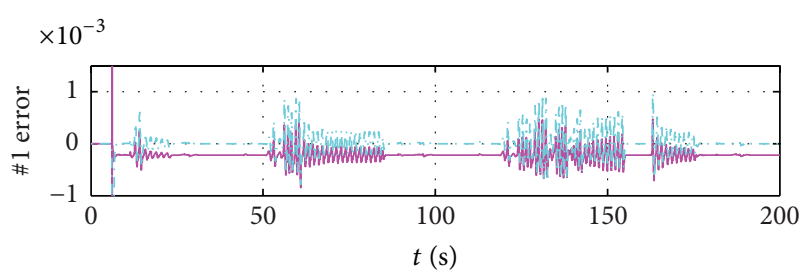

- Input observer

-.. Time delay observer

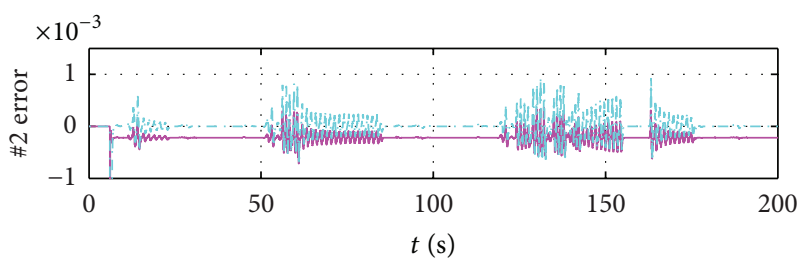

_ Input observer

-.. Time delay observer

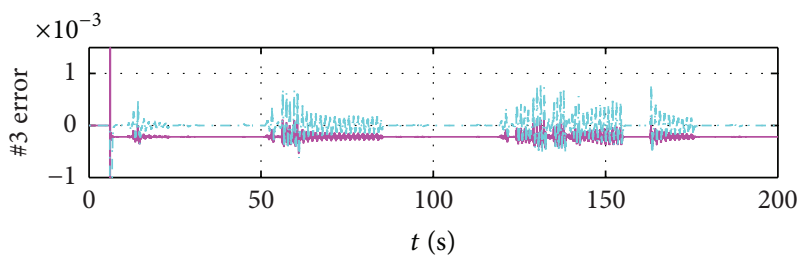

- Input observer

-.. Time delay observer

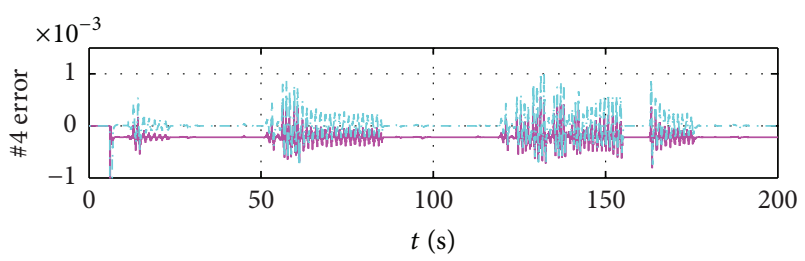

- Input observer

Time delay observer

FIGURE 7: Evolution of the estimation error of the proposed method and unknown input observer.

the individual cylinder AFR based on the proposed observer was presented. The performance of the proposed method was validated by the simulation data from engine software enDYNA provided by Tesis, and a comparison with existing method was obtained during ECE cycle, demonstrating that the proposed method considering time delay from exhaust gas transport and UEGO sensor dynamics can improve the accuracy of the individual cylinder AFR estimation.

\section{Competing Interests}

The authors declare that they have no competing interests.

\section{Acknowledgments}

This work was supported by China Automobile Industry Innovation and Development Joint Fund (no. U1564213).

\section{References}

[1] J. B. Heywood, Internal Combustion Engine Fundamentals, vol. 930, McGraw-Hill, New York, NY, USA, 1988.

[2] J. W. Grizzle, K. L. Dobbins, and J. A. Cook, "Individual cylinder air-fuel ratio control with a single EGO sensor," IEEE Transactions on Vehicular Technology, vol. 40, no. 1, pp. 280-286, 1991.

[3] J. Chauvin, P. Moulin, G. Corde, N. Petit, and P. Rouchon, "Realtime nonlinear individual cylinder air fuel ratio observer on a diesel engine test bench," in Proceedings of the 16th Triennial World Congress of International Federation of Automatic Control (IFAC '05), pp. 194-199, Prague, Czech Republic, July 2005.

[4] J. Chauvin, P. Moulin, G. Corde, N. Petit, and P. Rouchon, "Kalman filtering for real-time individual cylinder air fuel ratio observer on a diesel engine test bench," in Proceedings of the American Control Conference, pp. 1886-1891, IEEE, Minneapolis, Minn, USA, June 2006.

[5] J. Chauvin, N. Petit, and P. Rouchon, "Six degrees crankshaft individual air fuel ratio estimation of diesel engines for cylinder balancing purpose," Sae Technical Papers, 2006.

[6] Y. Liu and T. Shen, "Modeling and experimental validation of air-fuel ratio under individual cylinder fuel injection in gasoline engines," IEEJ Journal of Industry Applications, vol. 1, no. 3, pp. 155-163, 2012.

[7] J. F. Burkhard, "Individual cylinder fuel control for a turbocharged engine," SAE Technical Paper 0148-7191, SAE International, 2014.

[8] H. Li, Y. Huang, G. Li, and Y. Yang, "Research on the cylinderby-cylinder variations detection and control algorithm of diesel engine," SAE Technical Paper 0148-7191, 2015.

[9] S. Nakagawa, A. Numata, and T. Hori, "Individual cylinder control for air-fuel ratio cylinder imbalance," SAE Technical Paper 2015-01-1624, 2015.

[10] M. Kassa, C. Hall, A. Ickes, and T. Wallner, "In-cylinder oxygen mass fraction estimation method for minimizing cylinder-tocylinder variations," SAE Technical Paper 0148-7191, 2015.

[11] N. Qiao, C. Krishnamurthy, and N. Moore, "Determine air-fuel ratio imbalance cylinder identification with an oxygen sensor," SAE International Journal of Engines, vol. 8, no. 3, pp. 1005-1011, 2015.

[12] K. Suzuki, T. Shen, J. Kako, and S. Yoshida, "Individual A/F estimation and control with the fuel-gas ratio for multicylinder IC engines," IEEE Transactions on Vehicular Technology, vol. 58, no. 9, pp. 4757-4768, 2009.

[13] L. Benvenuti, M. D. Di Benedetto, S. Di Gennaro, and A. Sangiovanni-Vincentelli, "Individual cylinder characteristic estimation for a spark injection engine," Automatica, vol. 39, no. 7, pp. 1157-1169, 2003.

[14] J. Chauvin, G. Corde, P. Moulin, N. Petit, and P. Rouchon, "High frequency individual cylinder estimation for control of diesel engines," Oil and Gas Science \& Technology, vol. 61, no. 1, pp. 57-72, 2006.

[15] B. He, T. Shen, J. Kako, and M. Ouyang, "Input observer-based individual cylinder air-fuel ratio control: modelling, design and validation," IEEE Transactions on Control Systems Technology, vol. 16, no. 5, pp. 1057-1065, 2008.

[16] W.-A. Zhang and L. Yu, "Stability analysis for discrete-time switched time-delay systems," Automatica, vol. 45, no. 10, pp. 2265-2271, 2009. 
[17] S. P. Boyd, L. El Ghaoui, E. Feron, and V. Balakrishnan, Linear Matrix Inequalities in System and Control Theory, vol. 15, SIAM, 1994.

[18] TESIS DYNAware, en-DYNA ${ }^{\circledR}$ THERMOS ${ }^{\circledR} 2.0$ Block Reference Manual, 2006.

[19] T. DYNAware, en-DYNA ${ }^{\circledR}$ THERMOS ${ }^{\circledR} 2.0$ User Manual, 2006. 


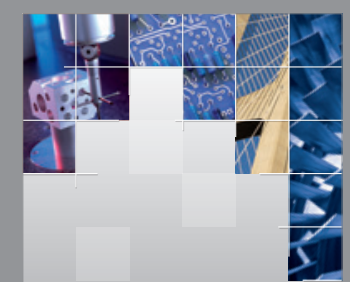

\section{Enfincering}
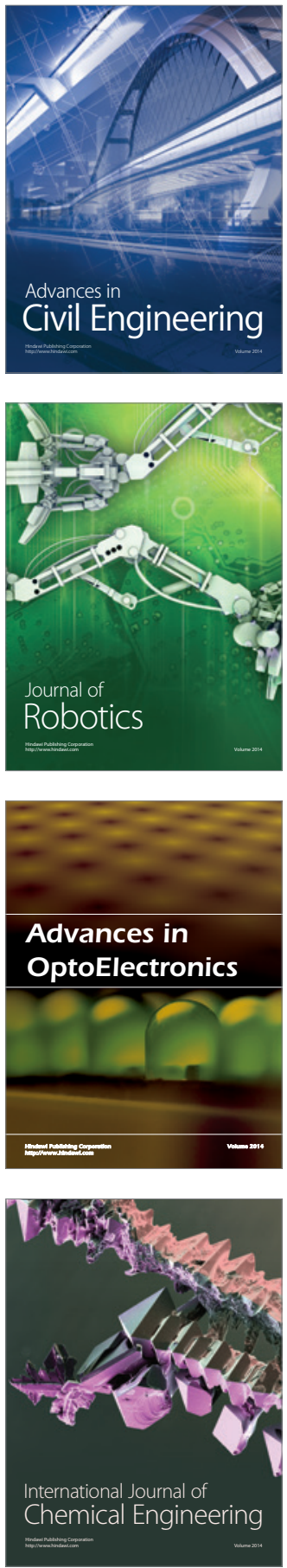

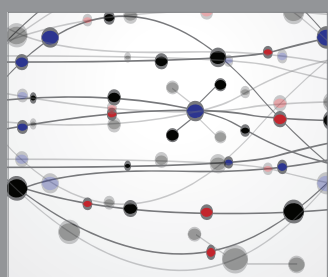

The Scientific World Journal

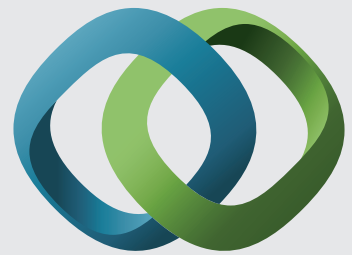

\section{Hindawi}

Submit your manuscripts at

http://www.hindawi.com
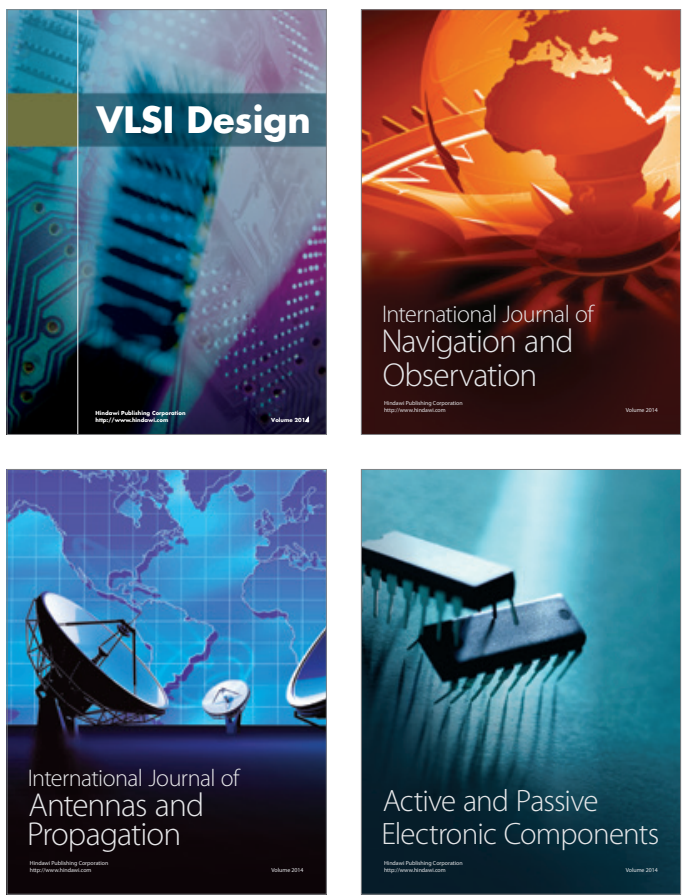
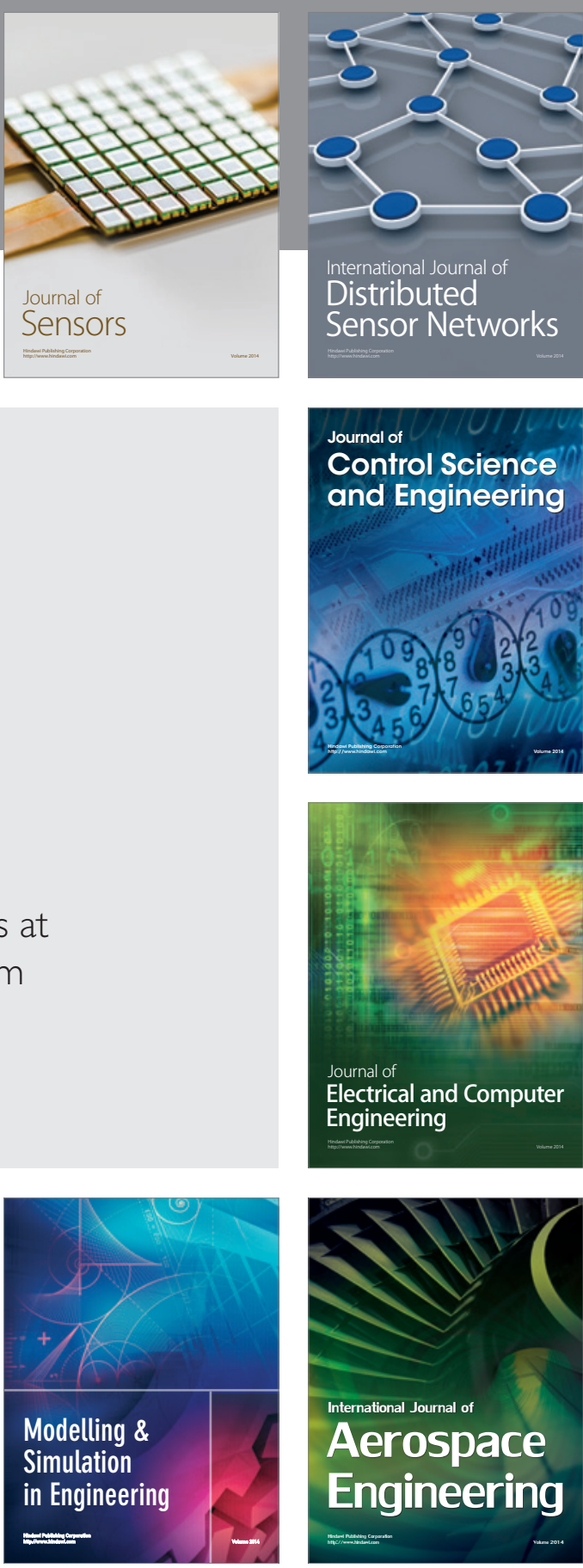

International Journal of

Distributed

Sensor Networks

Journal of

Control Science

and Engineering
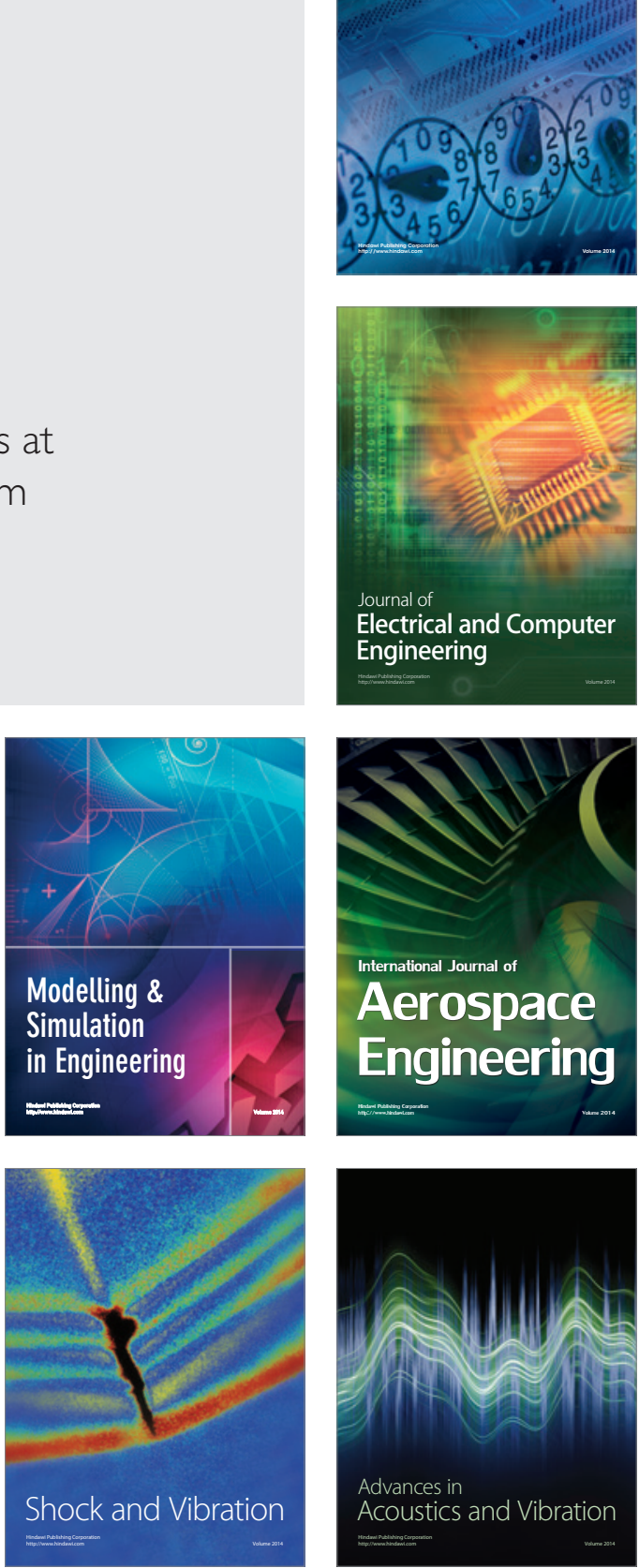